\title{
The Bonn-Gatchina analysis of the data from Kp collision reactions
}

Matveev M.A.

NRC "Kurchatov Institute", PNPI, Gatchina, Russia

E-mail: max.matveev.2010dgmai1.com

\section{Sarantsev A.V.*}

NRC "Kurchatov Institute", PNPI, Gatchina, Russia

HISKP, Bonn University, Bonn, Germany

E-mail: andsardhiskp.uni-bonn.de

In the framework of the Bonn-Gatchina formalism we perform the combined partial wave analysis of the data on $K p$ collision reactions. The obtained solution reveals signals from three previously unknown $\Sigma$-hyperon states. Two of these states are located in the mass region above $2 \mathrm{GeV}$ and one state with quantum numbers $J^{P}=1 / 2^{-}$in the mass region below $1.7 \mathrm{GeV}$.

XVII International Conference on Hadron Spectroscopy and Structure - Hadron2017

25-29 September, 2017

University of Salamanca, Salamanca, Spain

\footnotetext{
* Speaker.
} 


\section{Introduction}

The quark model predicts that baryons should form the quark multiplets: octets which consist of nucleons, $\Lambda, \Sigma$ and $\Xi$ particles and decuplets which consist of the $\Delta, \Sigma, \Xi$ and $\Omega$ particles. If observed nucleons and $\Delta$-resonances are three quark systems they should have partners in the hyperon sector. While nucleons can be only members of the octets the $\Lambda$-hyperons can also be singlet states. It means that in agreement with the quark model we should find more $\Lambda$ hyperons than the number of observed nucleons. The $\Sigma$ hyperons are members of octets and decuplets. Therefore one should expect the number of $\Sigma$-hyperons to be equal to the sum of observed nucleons and $\Delta$-states.

If a state can not be classified as a multiplet member it should be regarded as an exotic state from the point of view of the quark classification. Therefore it is very important to define the multiplet members in both the non-strange and strange-quark sectors. If the number of known $\Lambda$ states is indeed larger than the number of 4-star nucleon states the situation in the $\Sigma$-hyperon sector is challenging. For example, the only one $1 / 2^{-} \Sigma$-hyperon was observed with the mass below 1.9 $\mathrm{GeV}$. However we should expect at least three states: the multiplet partners of the $N(1535) 1 / 2^{-}$, $N(1620) 1 / 2^{-}$and $\Delta(1620) 1 / 2^{-}$. It is very important to understand whether we observe a violation of the quark model or there are missing states which escaped the identification in the previous data analyses.

Practically all information about spectra and properties of hyperons was obtained from the analysis of $K p$ collision reactions with production of two particles in the final state. Moreover most of results come from the data on the differential cross section. For reactions with nucleons in the final state the polarization observables were not measured. The recoil polarization is available from experiments with hyperons in the final state, for example $K p \rightarrow \pi^{0} \Lambda$. However one needs to measure at least three observables: the differential cross section, the target (or recoil) asymmetry and the spin rotation parameter to obtain the unambiguous partial wave decomposition at fixed energies. Moreover these observables should be measured with a good precision and the full angular coverage. Therefore we could expect that the analysis of the data from one experiment can miss some signals due to ambiguities in the solution.

The problem mentioned above can be successfully solved in the combined energy-dependent partial wave analysis of all available data. In this case the basic properties of the amplitude such as unitarity and analyticity provide a strong constraint to the solution. Such approach was successfully applied by our group to the analysis of the meson pion-induced and photo-production data. Recently the ANL-Osaca group applied a similar approach to the analysis of the $K p$ collision reactions with two particles in the final state [四]. In this paper we present the result of our analysis of these data taken in the mass rage from the thresholds up to $2.3 \mathrm{GeV}$.

\section{Fit of the data}

As the starting point we included in the fit all resonances from PDG with 3 and 4-star rating as non-relativistic Breit-Wigner states. Such approach allows us to fix the parameters of the fitted states within the PDG errors. Although we were able to obtain an acceptable descriptions of the data practically at all masses, the fit showed systematical deviations in the region $1.7 \mathrm{GeV}$ for the 
$K p \rightarrow \pi^{+} \Sigma^{-}$and $K p \rightarrow \pi^{-} \Sigma^{+}$differential cross section. This is clearly seen in the comparison of the total cross section calculated from the data and the fit (see dashed curves in Fig. W). At high masses there are also some deviations between the data and the fit for the $K p \rightarrow \pi \Lambda$ and all $K p \rightarrow \pi \Sigma$ reactions. To understand whether such deviations can be due to missing states we have added in all partial waves one by one the $\Lambda$ and $\Sigma$ resonances with fixed mass and performed the mass scans of the introduced states. In the $\Lambda$ sector we observed only marginal improvements from the new states. The effect from the $\Sigma$-hyperons is shown in Fig. $\square$. We observe three rather promising minima in the $1 / 2^{-}$partial wave and the minima in the $5 / 2^{-}, 7 / 2^{-}, 3 / 2^{+}$and $1 / 2^{+}$ partial waves.

The most prominent signal was found in the $1 / 2^{-}$partial wave. The added resonance optimized with the mass $1660 \mathrm{MeV}$ and width $220 \mathrm{MeV}$. Then we added to this solution one additional state and scanned its mass. The new scans did not find the clear minima for the $\Sigma\left(1 / 2^{+}\right)$partial wave and only show one minimum in the $\Sigma\left(1 / 2^{-}\right)$partial wave, located above $2 \mathrm{GeV}$. Other quantum numbers in the $\Sigma$-sector still showed prominent minima, but for $3 / 2^{+}$the minimum became a notably weaker. After including in the fit the $5 / 2^{-}$state and again repeating mass scans we have found only very small minima for $7 / 2^{-}$and $3 / 2^{+}$partial wave. The final solution included two new $1 / 2^{-}$states (the second one is in the mass region just above $2.1 \mathrm{GeV}$ ) and one $5 / 2^{-}$state in the mass region above $2.2 \mathrm{GeV}$. The solution produced the best description of the data and was stable against adding new states to the fit.
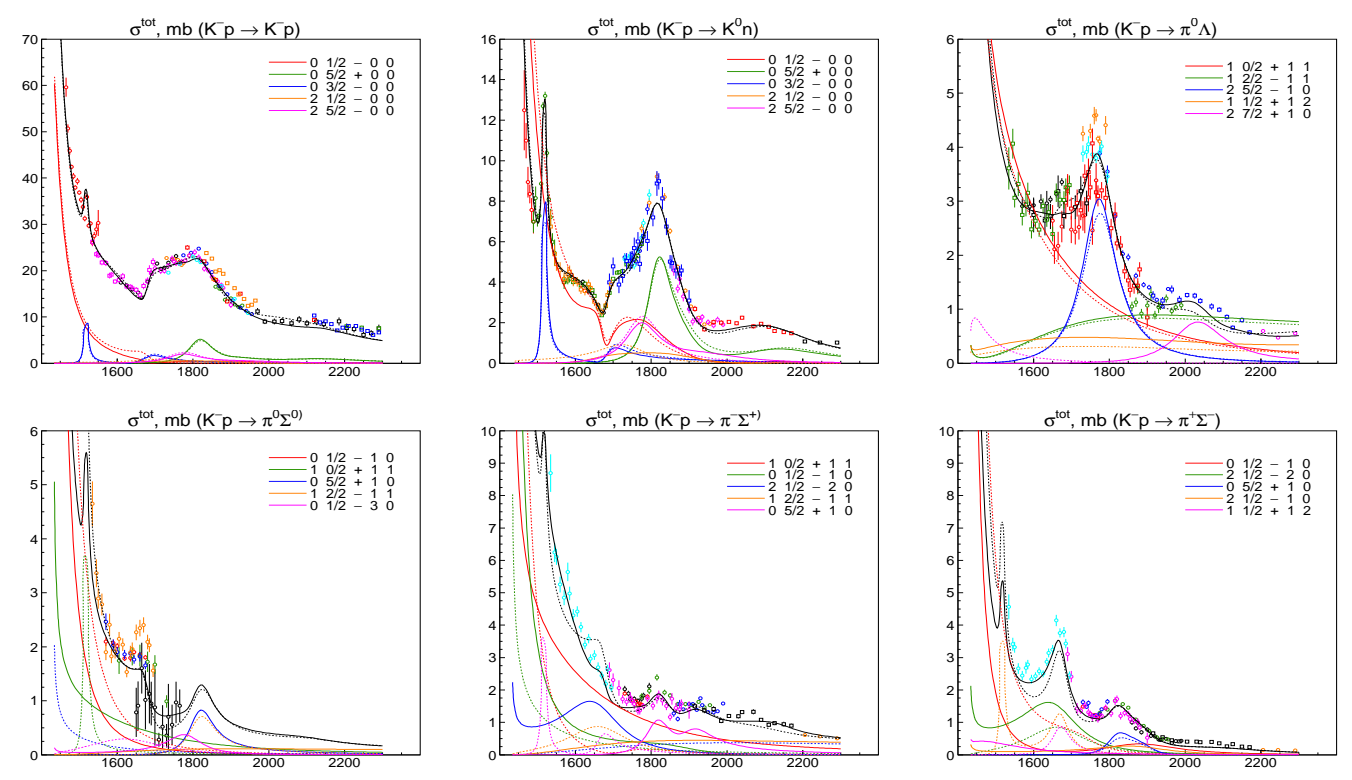

Figure 1: (Color online) The comparison of the total cross section calculated from the data and from the two fits. Dashed curves show the fit with resonances from PDG rated with 3 and 4 stars. Full curves show the solution with added three new $\Sigma$-states. The data are taken from [D]-[ㄹ]

To impose the unitarity condition to the fitted amplitudes we, at first, substituted the nonrelativistic Breit-Wigner states with the relativistic ones. This imposed the important restriction on the resonance couplings: the couplings provide directly the partial widths of the state and the sum of them can not exceed the total width. As the next step we introduced the K-matrix parameterization 
Table 1: The list of the fitted reaction and the comparison of the our description (Bn-Ga) with descriptions from the two solutions found in the ANL-Osaca approach [四].

\begin{tabular}{|l|crcccc|}
\hline & & ANL-Osaca & Bn-Ga & Model A & Model B & Bn-Ga \\
\hline & & N(data) & N(data) & $\chi / N$ & $\chi / N$ & $\chi / N$ \\
\hline$K^{-} p \rightarrow K^{-} p$ & $d \sigma / d \Omega$ & 3962 & 5495 & 3.07 & 2.98 & 2.28 \\
& $P$ & 510 & 859 & 2.04 & 2.08 & 1.79 \\
\hline$K^{-} p \rightarrow \bar{K}^{0} n$ & $d \sigma / d \Omega$ & 2950 & 3445 & 2.67 & 2.75 & 1.62 \\
\hline$K^{-} p \rightarrow \pi^{-} \Sigma^{+}$ & $d \sigma / d \Omega$ & 1792 & 2095 & 3.37 & 3.49 & 3.17 \\
& $P$ & 418 & 578 & 1.30 & 1.28 & 2.06 \\
\hline$K^{-} p \rightarrow \pi^{0} \Sigma^{0}$ & $d \sigma / d \Omega$ & 580 & 581 & 3.68 & 3.50 & 3.57 \\
& $P$ & 196 & 124 & 6.39 & 5.80 & 1.51 \\
\hline$K^{-} p \rightarrow \pi^{+} \Sigma^{-}$ & $d \sigma / d \Omega$ & 1786 & 2082 & 2.56 & 2.18 & 1.80 \\
\hline$K^{-} p \rightarrow \pi^{0} \Lambda$ & $d \sigma / d \Omega$ & 2178 & 2478 & 2.59 & 3.71 & 1.82 \\
& $P$ & 693 & 732 & 1.41 & 1.73 & 1.73 \\
\hline
\end{tabular}

which automatically satisfies the unitarity condition and takes into account the rescattering between states with the same quantum numbers.

In the K-matrix solution, the low mass states have very small missing inelasticity. This is a very strong support for the obtained solution: the missed inelasticity can be only due to channels with the two pion production and the two-pion-hyperon cross section is rather small below $1.7 \mathrm{GeV}$.

Although new states improve the description of all data one can see a very significant improvement in the region $1.7 \mathrm{GeV}$ for the recoil asymmetry in the $K p \rightarrow \pi \Lambda$ reaction shown in Fig. B 3 and

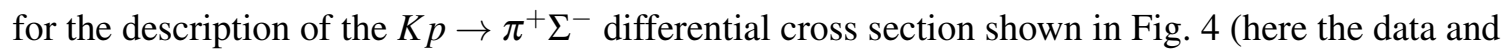
curves are shown in the logarithmic scale). Moreover the solution with three new resonances has dramatically different partial wave contributions in the mass region $1.7 \mathrm{GeV}$ than the fit with the PDG resonances only. For example the new state at $1660 \mathrm{MeV}$ strongly contributes to the $K p \rightarrow \pi \Sigma$ cross section (see Fig. 四).

The new high mass resonances provide a notable improvement practically in all fitted reactions with pion-hyperon final states (see the description of the data from Figs. [1, [1, (d). In most cases our fit provides a better description of the data than the two solutions reported in [四] although we have fitted a larger data set: we have selected the data up to the mass $2.3 \mathrm{GeV}$ while ANL-Osaca used only the data up to the mass $2.1 \mathrm{GeV}$. The comparison of our result and ANL-Osaca solutions is

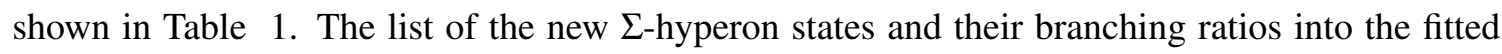
channels are given in Table $\square$

\section{Conclusion}

In the framework of the Bonn-Gatchina approach we have performed the combined energy dependent analysis of the $K p$ collision reactions with the two particles in the final states. The analysis reveals the signals from three previously unknown states $\Sigma(1660) 1 / 2^{-}, \Sigma(2160) 1 / 2^{-}$and 

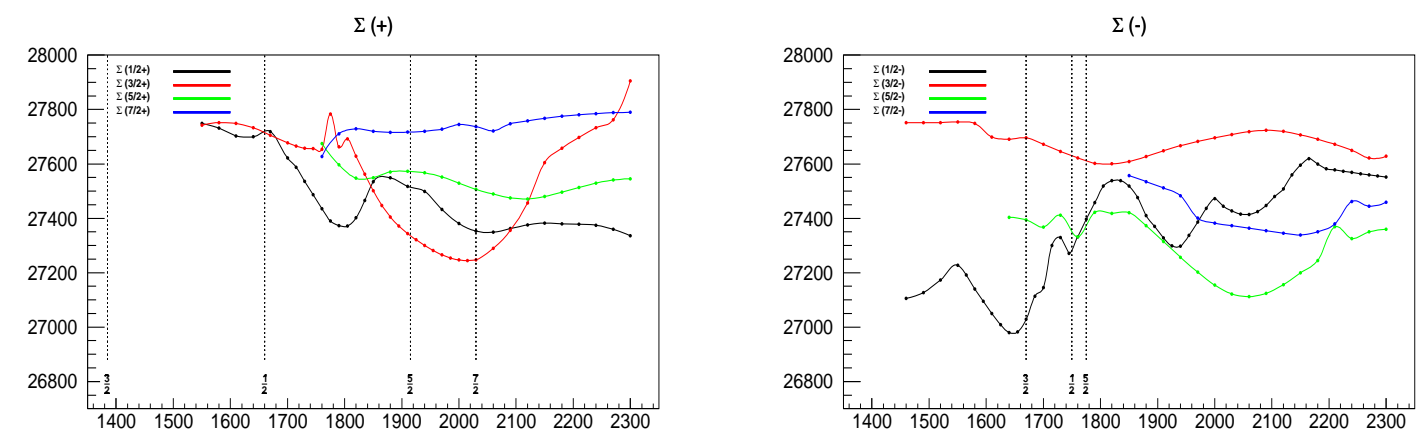

Figure 2: The change of the the $\log L$ value (Y-axis) when the mass of the introduced state is scanned over the fitted mass range.

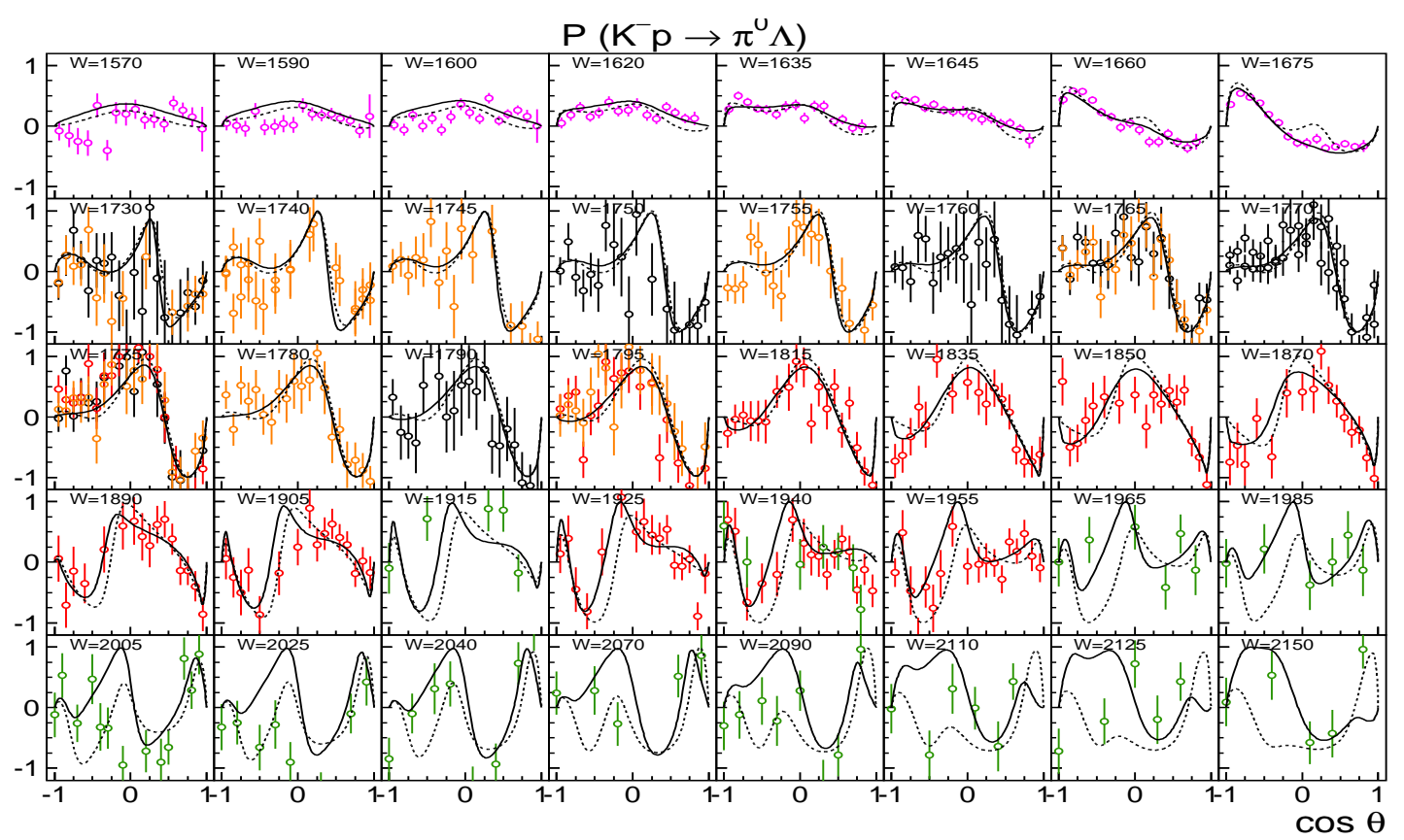

Figure 3: The description of the $K p \rightarrow \pi^{0} \Lambda$ recoil asymmetry. Dashed curves show the description of the data with resonances from PDG rated with 3 and 4 stars. Full curves show the description with the solution

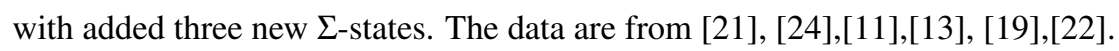

Table 2: The list of the found resonances and thier branchings to the fitted channels.

\begin{tabular}{|l|ccc|}
\hline & $\Sigma(1660) 1 / 2^{-}$ & $\Sigma(2160) 1 / 2^{-}$ & $\Sigma(2260) 5 / 2^{-}$ \\
\hline Mass $(\mathrm{MeV})$ & 1660 & 2160 & 2260 \\
Width(MeV) & 218 & 223 & 360 \\
$K p(\%)$ & 35 & 33 & 9 \\
$\pi \Lambda(\%)$ & 10 & 35 & 24 \\
$\pi \Sigma(\%)$ & 55 & 15 & 4 \\
\hline
\end{tabular}




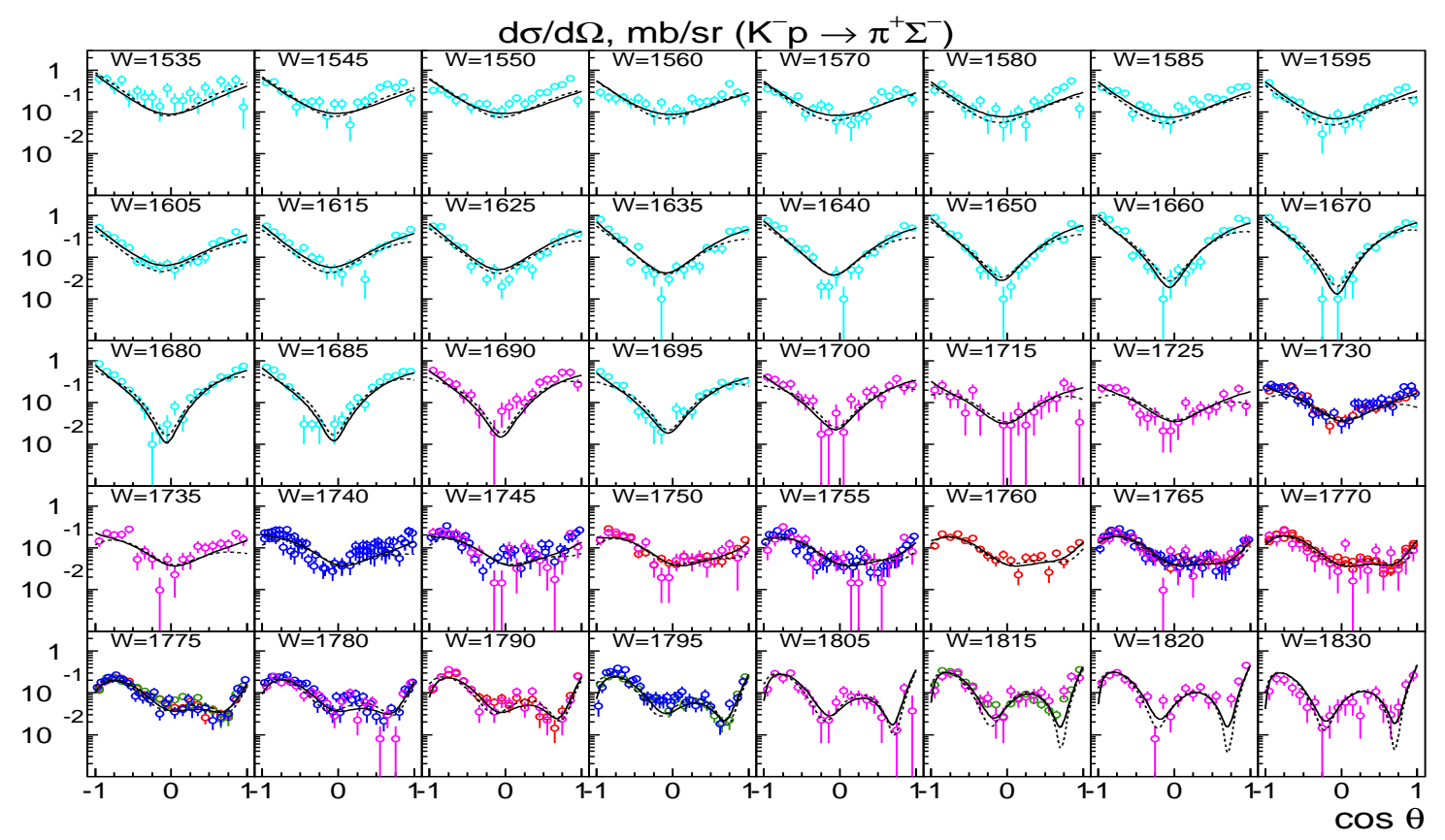

Figure 4: The description of the $K p \rightarrow \pi^{+} \Sigma^{-}$differential cross section at low energies. Dashed curves show the description of the data with resonances from PDG rated with 3 and 4 stars. Full curves show

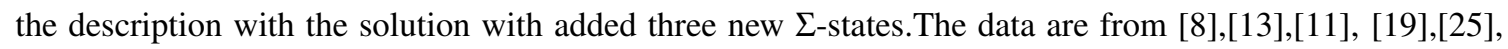
[ए2], [प4]], [ए]] .

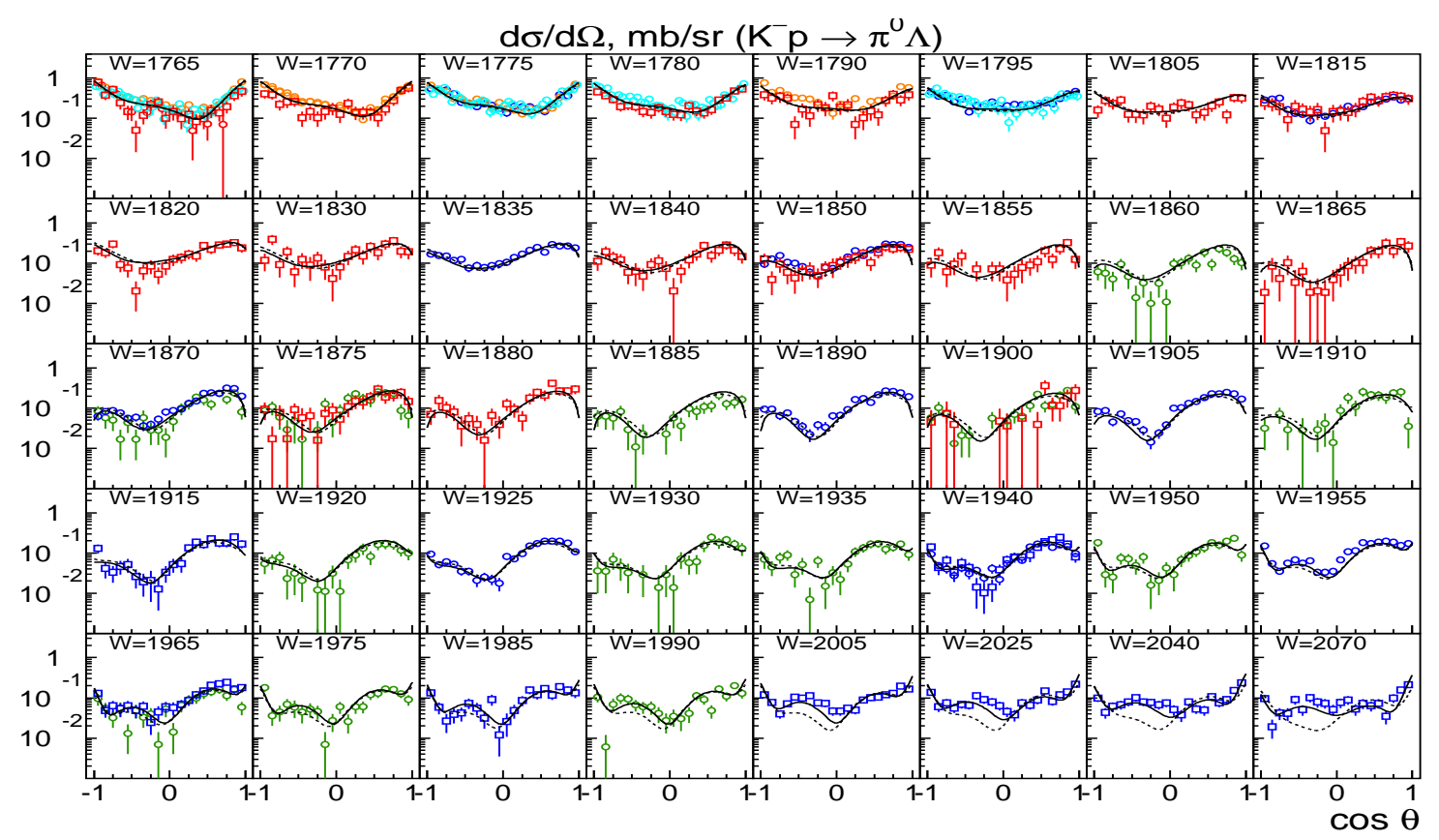

Figure 5: The description of the $K p \rightarrow \pi^{0} \Lambda$ cross section. Dashed curves show the description of the data with resonances from PDG rated with 3 and 4 stars. Full curves show the description with the solution with

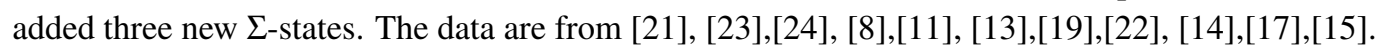


$\Sigma(2260) 5 / 2^{-}$. The first state can be considered as the multiplet partner for the well known nucleon resonance $N(1535) 1 / 2^{-}$.

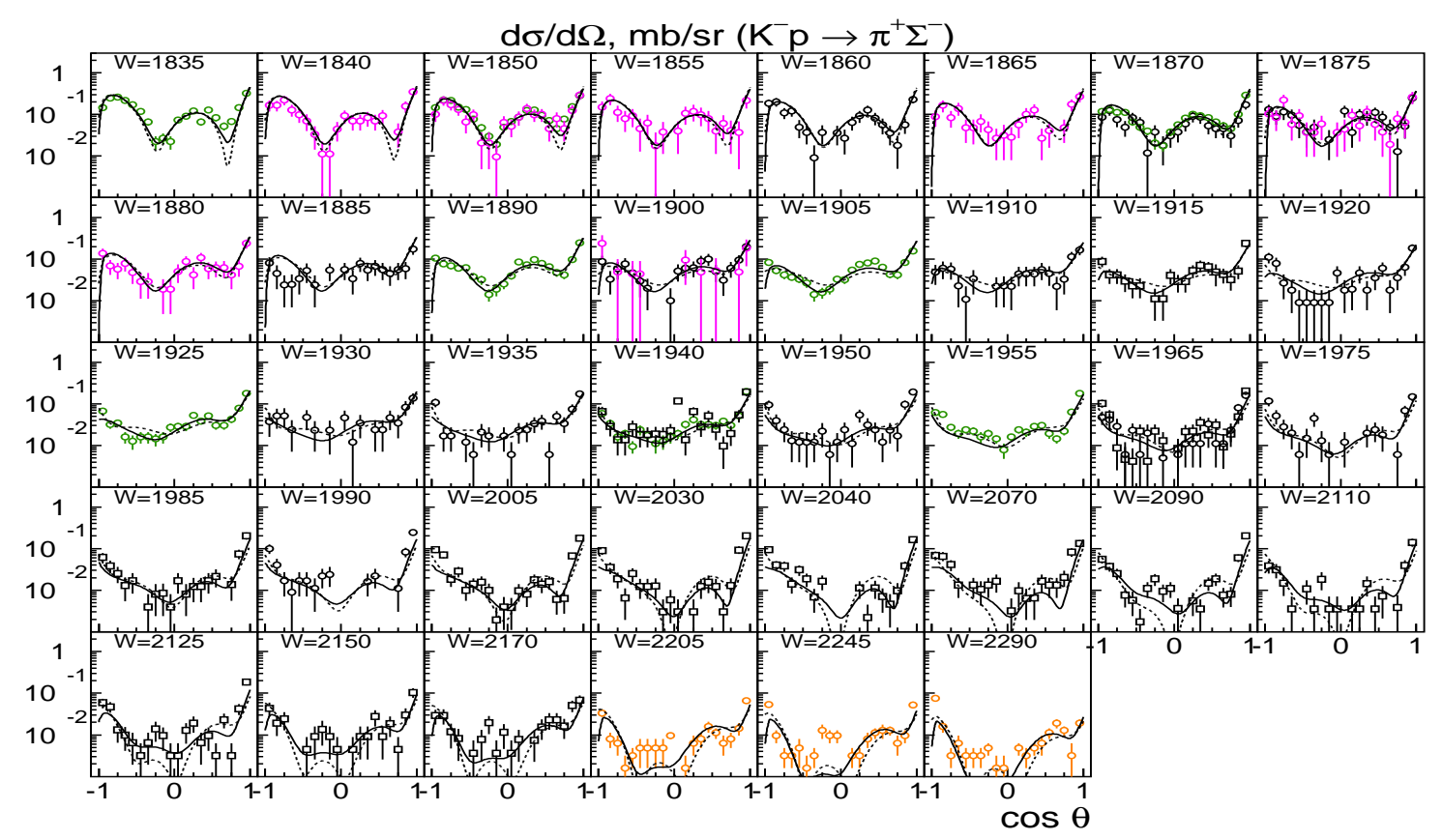

Figure 6: The description of the $K p \rightarrow \pi^{0} \Lambda$ recoil asymmetry. Dashed curves show the description of the data with resonances from PDG rated with 3 and 4 stars. Full curves show the description with the solution

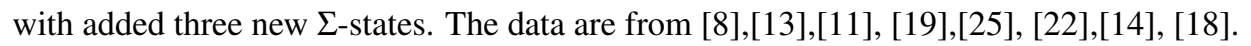

\section{Acknowledge}

This work was supported by the RSF grant 16-12-10267.

\section{References}

[1] H. Kamano, S. X. Nakamura, T.-S. H. Lee and T. Sato, Phys. Rev. C 90, no. 6, 065204 (2014) doi:10.1103/PhysRevC.90.065204 [arXiv:1407.6839 [nucl-th]].

[2] C. Daum, F. C. Erne, J. P. Lagnaux, J. C. Sens, M. Steuer and F. Udo, Nucl. Phys. B 6, 273 (1968).

[3] S. Andersson-Almehed, C. Daum, F. C. Erne, J. P. Lagnaux, J. C. Sens and F. Udo, Nucl. Phys. B 21, 515 (1970).

[4] M. G. Albrow, S. Andersson-Almehed, B. Bosnjakovic, F. C. Erne, Y. Kimura, J. P. Lagnaux, J. C. Sens and F. Udo, Nucl. Phys. B 29, 413 (1971).

[5] P. J. Litchfield et al., Nucl. Phys. B 30, 125 (1971).

[6] B. Conforto et al., Nucl. Phys. B 34, 41 (1971).

[7] P. C. Barber et al., Nucl. Phys. B 92, 391 (1975).

[8] J. Griselin et al., Nucl. Phys. B 93, 189 (1975). 
[9] T. S. Mast, M. Alston-Garnjost, R. O. Bangerter, A. S. Barbaro-Galtieri, F. T. Solmitz and R. D. Tripp, Phys. Rev. D 14, 13 (1976).

[10] P. C. Barber et al., Nucl. Phys. B 102, 365 (1976).

[11] B. Conforto et al. [Rutherford-London Collaboration], Nucl. Phys. B 105, 189 (1976).

[12] A. de Bellefon et al., Nuovo Cim. A 42, 403 (1977).

[13] W. Cameron et al. [Rutherford-London Collaboration], Nucl. Phys. B 193, 21 (1981).

[14] R. Armenteros et al., Nucl. Phys. B 21, 15 (1970).

[15] G. W. London et al., Nucl. Phys. B 85, 289 (1975).

[16] C. J. Adams et al., Nucl. Phys. B 96, 54 (1975).

[17] A. Berthon et al., Nucl. Phys. B 20, 476 (1970).

[18] A. Berthon et al., Nucl. Phys. B 24, 417 (1970).

[19] M. Jones, R. Levi Setti, D. Merrill and R. D. Tripp, Nucl. Phys. B 90, 349 (1975).

[20] M. Alston-Garnjost, R. W. Kenney, D. L. Pollard, R. R. Ross, R. D. Tripp and H. Nicholson, Phys. Rev. D 17, 2226 (1978).

[21] S. Prakhov et al., Phys. Rev. C 80, 025204 (2009).

[22] R. Armenteros et al., Nucl. Phys. B 8, 233 (1968).

[23] D. F. Baxter et al., Nucl. Phys. B 67, 125 (1973).

[24] A. de Bellefon et al., Nuovo Cim. A 90, 1 (1975).

[25] A. de Bellefon et al., Nuovo Cim. A 37, 175 (1977).

[26] R. D. Ehrlich, B. Lovett, M. Mishina, P. A. Souder, J. Snyder, M. E. Zeller, D. M. Lazarus and I. Nakano, Phys. Lett. 71B, 455 (1977).

[27] H. C. Bryant et al., Nucl. Phys. B 168, 207 (1980). 